\title{
A Fuzzy Logic based Model for Predicting Commercial Banks Financial Failure
}

\author{
Ahmed A. Mohamed \\ Computers and Information Systems Department \\ Higher Technological Institute \\ Cairo, Egypt
}

\author{
Ahmed S. Salama \\ Computer and Information Systems Department \\ Sadat Academy for Management Sciences (SAMS) \\ Cairo, Egypt
}

\begin{abstract}
The financial failure of most commercial banks is hardly identified and detected. Machine learning techniques as Fuzzy logic (FL), Artificial Neural network (ANN), Case based reasoning (CBR) and Rule based system (RBS) are important techniques that are used in prediction and forecasting generally. This paper introduces a fuzzy logic based proposed model which can help the decision maker in commercial banks to make the right decision to determine level of financial failure in the bank. The proposed model employing financial ratios rules used in Egyptian commercial banks to measure the bank financial performance indicators i.e. capital adequacy, asset quality, liquidity, and earnings in order to determine their financial failure. The proposed model uses fuzzy logic in MATLAB to build financial ratios membership functions. In addition, the implementation of the proposed model used visual studio 2010 to build the graphical user interface for the user. The implemented system was tested on two of the Egyptian commercial banks i.e. CIB bank and CFEB bank. The mathematical equation of the defuzzification was applied to calculate financial failure in these two banks. After implementing and applying the proposed model, it was proved that the fuzzy logic technique is one of the most important machine learning techniques that can be used to detect financial failure in commercial banks and the proposed model proved that it is highly effective, scalable, and reliable in detecting the financial bank failure.
\end{abstract}

\section{General Terms}

Machine Learning, Fuzzy Logic

\section{Keywords}

Machine Learning, Fuzzy Logic, Defuzzification, Financial Ratios, Financial Prediction.

\section{INTRODUCTION}

Banks are the most important financial institutions in Egypt. The Egyptian banks have an experience in financial difficulties since many substantial banks failed in the past. This event urged the government to take measures to prevent banks from getting into financial difficulties. From year to year, strong attention has been paid to the study of the problems of predicting and preventing bank from bankruptcy. Particularly after the world financial crisis had occurred, the depositors and investors withdrew their money from the banks because they were afraid of bankruptcy of the banks, in addition they don't know what will happen to the banks in the future. The failure of the banks affects directly the economy and many industries. The failure of the banks has many reasons. This study will propose a model that clarifies the direct reasons for failure of the banks. It will also predict the failure of the banks before taking place and it will help the government and banks develop a quick remedy to prevent this failure. The model developed in this paper can be used to assist the concerned parties in predicting the probability of financial failure of the commercial Banks. Bank managers can use this model to assess the likelihood of failure. If there is possibility of failure, they can take the necessary decisions in advance. Investors can use this model to assess the financial health of the banks that they are concerned with. This research focuses on the probability of failure of commercial banks in Egypt. The purpose of this paper is to provide and develop early warning system for predicting banks financial failure.

\section{LITERATURE REVIEW}

In general, several approaches have been used in the predicting models, such as machine learning to establish a model capable of detecting the failure of the banks and classify the cases of failure and success of banks.

Edward I. Altman in 1997 conducted a study to Predict Corporate Bankruptcy using Discriminant Analysis.[1] James Kolari, Dennis Glennon, Hwan Shin, and Michele Caputo in 2000 conducted a study to predict the failure of the commercial bank in U.S. Because of sampling limitations, research has examined the feasibility of using computer-based early warning systems to make such Predictions. [2] Laitinen, E.K. and T. Laitinen in 2000 conducted a study to predict the failure of the commercial banks using logistic regression. [3] Molina, C. A. in 2002 conducted a study to develop Models that predict financial failure in USA using a simple dynamic hazard model. [4] M.Mete Doğanay, Nildağ Başak Ceylan, Ramazan Aktas in 2005 conducted a study to develop Models that predict financial failure in the Turkish banks and used discriminate analysis and Multiple Linear Regression to predict the probability of financial failure of the Turkish Banks.[5] Gleb Lanine; Rudi Vander Vennet in 2007 conducted a study to predict the failure of the Russian commercial banks using logit and trait recognition models. [6] Birsen Eygi Erdogan in 2008 conducted a study to predict the failure of the Turkish commercial banks. Logistic Regression was used to form a prediction model with financial ratios. 42 commercial banks were included in this research. [7] Maisarh, Yaseen; Omar in 2008 conducted a study to predict the failure of the Yemeni banks using logistic regression, discriminant analysis and Multiple Linear Regression.[8] Nedaa Mohammed ezzat in 2010 conducted a study exploiting machine learning in generating future scenarios for the Tourism Industry in Egypt and used neural network to introduce scenarios for the Tourism Industry. [9] Petr Dostil used the fuzzy logic for prediction of the time series. He came to a conclusion that the fuzzy logic helps in decentralization of decision-making processes to be standardized, reproduced, and documented, that is an important factor in the business field[10]. 


\section{THE PROPOSED MODEL ARCHITECTURE}

components of the proposed model are discussed briefly in the following subsections.

The proposed model architecture of the commercial banks financial failure detection is shown in Figure 1. The main

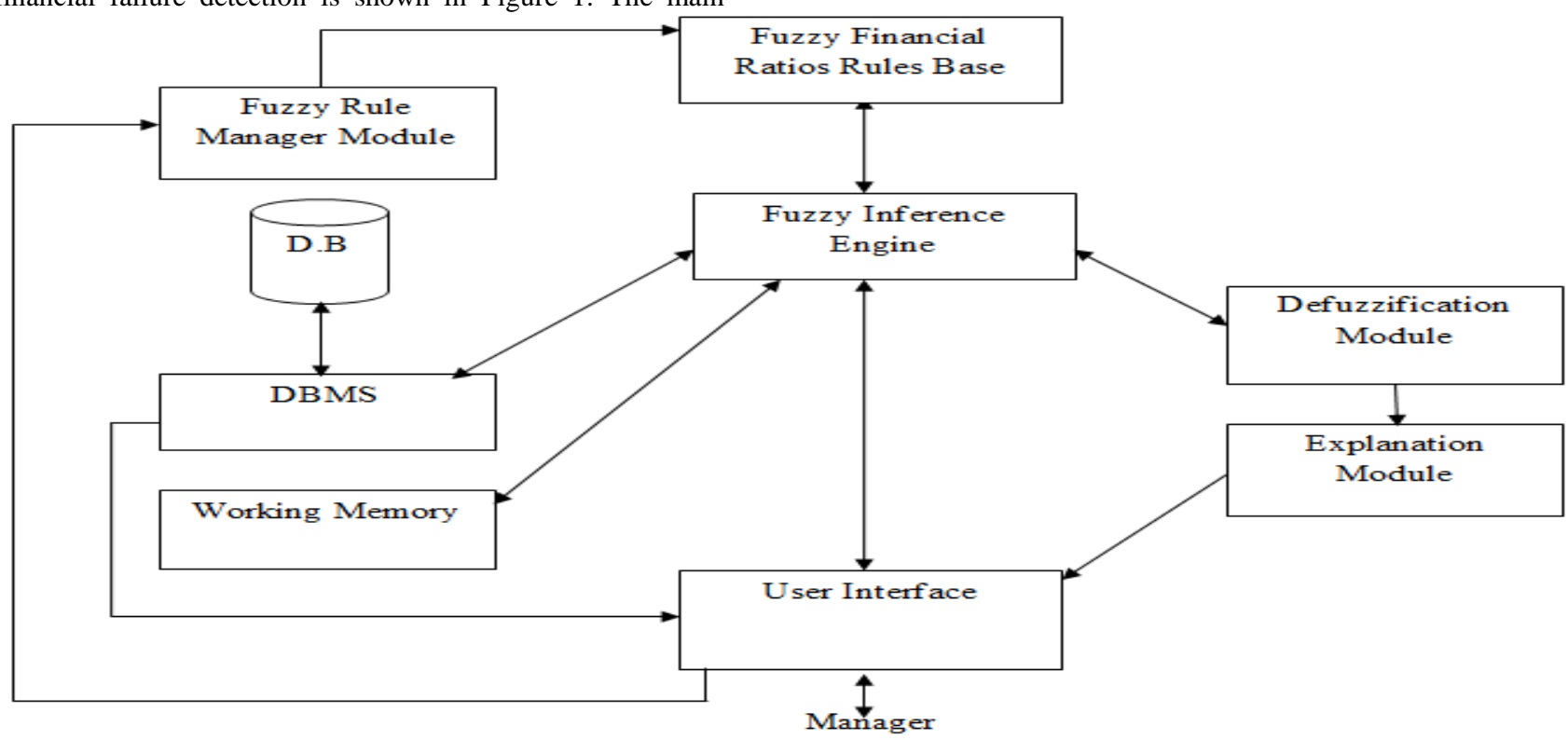

Fig 1: The Architecture of Proposed Model

\subsection{Fuzzy Financial Ratios Rules Base}

Financial ratios rule base consists of fact and fuzzy rules. The model is designed to be able to add, or update these rules in case of expanding the financial rules base. Fuzzy rules are used to measure financial ratios such as capital adequacy, asset quality, earnings and liquidity. The financial ratios are classified into four categories as follows:

\subsubsection{Capital Adequacy Ratio}

Capital adequacy ratio is used to predict financial failure, and it is based on the capital adequacy indicator.

a- Capital Adequacy indicator

$\mathrm{EA}=$ Equities / Assets

b- Capital Adequacy rules

This section specifies the capital adequacy ratio rules of the fuzzy inference module which are presented in Table 1 . The certainty factor $(\mathrm{CF})$ is calculated to each rule according to the value which was determined by the CBE (Central Bank of Egypt) and the linguistic variables table.

Table 1. Capital adequacy rules

\begin{tabular}{|l|l|}
\hline Rule \# & \\
\hline 1 & IF $\quad$ EA is low \\
& THEN $\quad$ Capital Adequacy is high \\
& CF $(\mathrm{H}, \mathrm{E} 1)=$ min [4.8]= 4.8 \\
\hline 2 & IF $\quad$ EA is medium \\
& THEN $\quad$ Capital Adequacy is medium \\
& CF $(\mathrm{H}, \mathrm{E} 1)=$ min [5.9]=5.9 \\
\hline 3 & IF $\quad$ EA is high \\
& THEN $\quad$ Capital Adequacy is low \\
& CF $(\mathrm{H}, \mathrm{E} 1)=$ min [6.7]=6.7 \\
\hline
\end{tabular}

\subsubsection{Asset Quality Ratios}

Asset quality ratios are used to predict financial failure, and it is based on the following four indicators (LPL, PA, LPSTL, and AFCLFC). a- $\quad$ Asset Quality Ratio indicators

- $\quad$ LPL = Loan provisions / Loans

- $\quad$ PA $=$ Provisions $/$ Assets

- $\quad$ LPSTL = Loans to Private Sector / Total Loans

- $\mathrm{AFCLFC}=$ Assets in Foreign Currencies / Liabilities in Foreign Currencies

b- Asset Quality Rules

This section specifies samples of the asset quality ratio rules of the fuzzy inference module which are presented in table 2 .

Table 2. Asset Quality Rules Samples

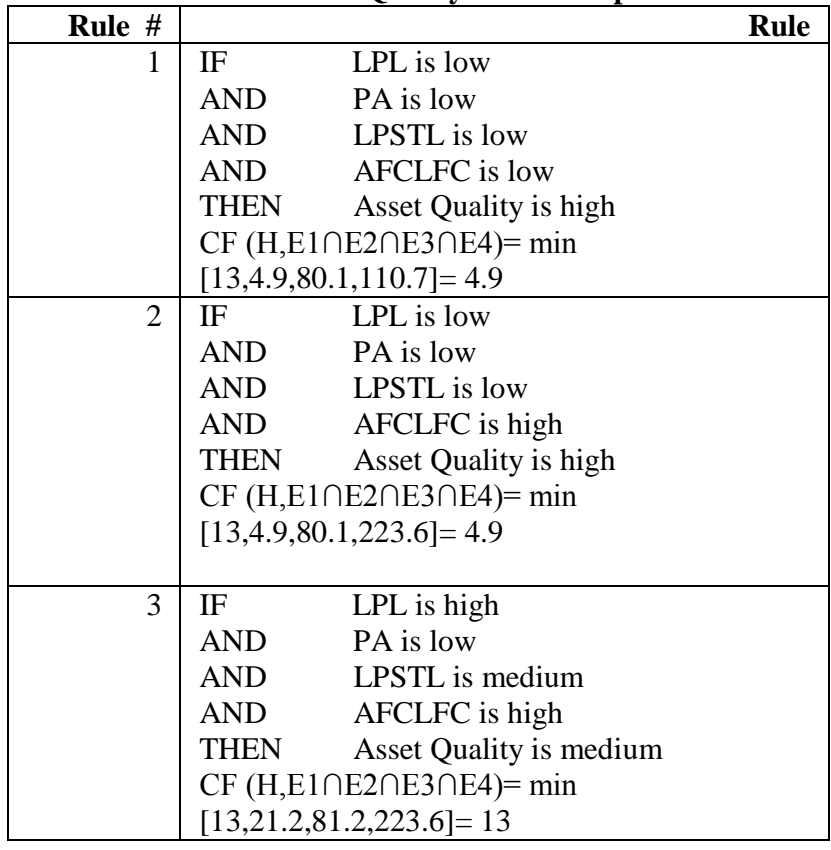




\subsubsection{Earnings Ratios}

Earnings ratios are used to predict financial failure, and it is based on calculating the following four indicators (NIIA, NOIIA, NPE, and NPA).

a- Earnings Indicators

- $\quad$ NIIA $=$ Net interest income $/$ Assets

- $\quad$ NOIIA $=$ Non - interest income $/$ Assets

- $\quad$ NPE $=$ Net profit / Equities

- $\quad$ NPA $=$ Net profit / Assets

b- Earnings Ratios Rules

This section specifies samples of the earnings ratios rules of the fuzzy inference module which are presented in table 3 .

Table 3. Earnings Rules Samples

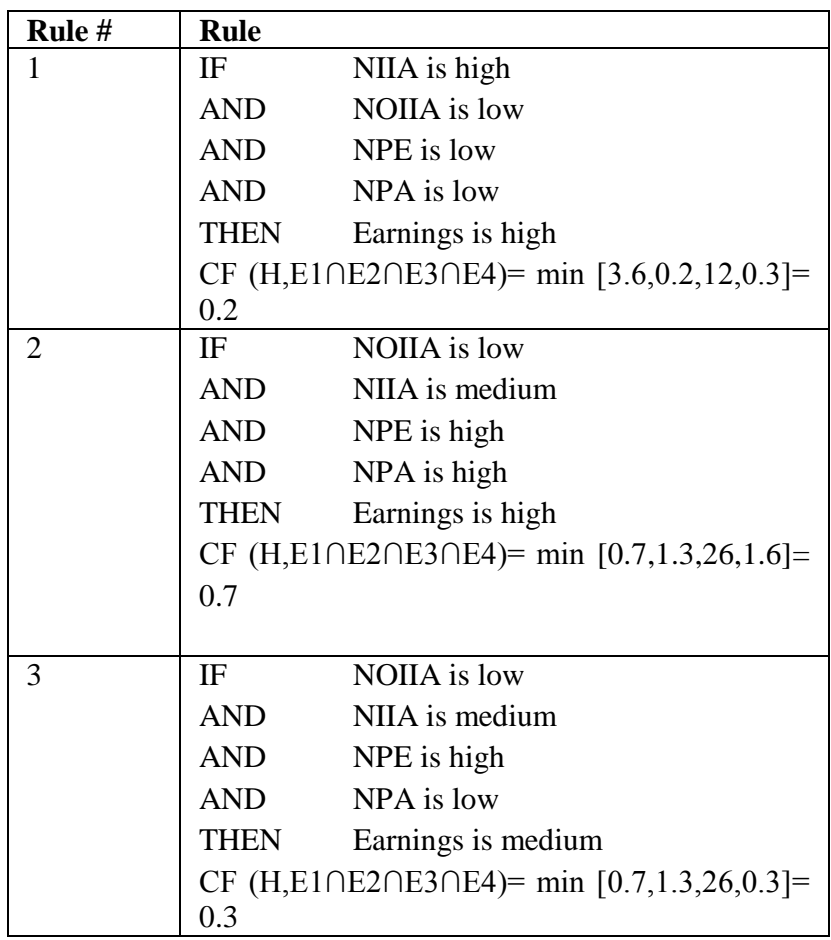

\subsubsection{Liquidity Ratios}

Liquidity ratios are used to predict financial failure, and they are based on calculating the following four indicators (SA, $\mathrm{DA}, \mathrm{LD}$, and ARRB).

$$
\begin{aligned}
\text { a- } & \text { Liquidity Indicators } \\
\text { - } & \text { SA }=\text { Securities } / \text { Assets } \\
\text { - } & \text { DA = Deposits / Assets } \\
\text { - } & \text { LD }=\text { Loans / Deposits } \\
\text { - } & \text { ARRB = Actual Reserve Ratio at Banks }
\end{aligned}
$$

b- Liquidity Ratios Rules

This section specifies samples of the liquidity ratios rules of the fuzzy inference module which are presented in table 4
Table 4. Liquidity Rules Samples

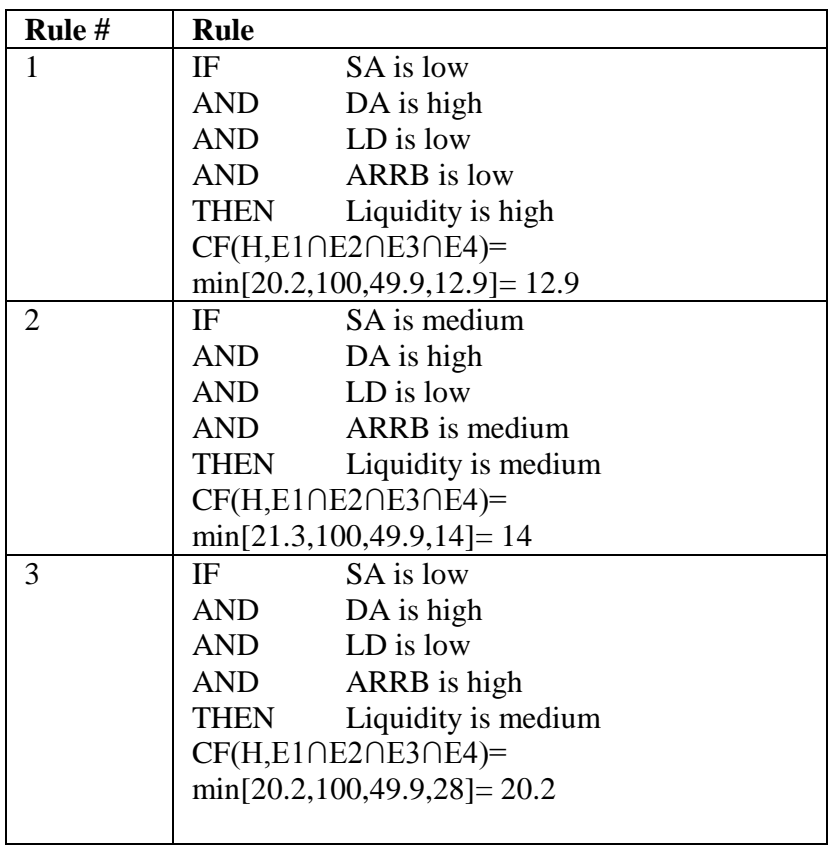

\subsection{Fuzzy Inference Engine}

Fuzzy inference engine is used as the core of the reasoning process. The fuzzy inference process is based on four steps:[11]

a. Fuzzification of the input variables

b. Rule evaluation

c. Aggregation of the rule outputs

d. Defuzzification

As discussed earlier, the inputs of the system consists of the indicators values for the capital adequacy, asset quality, earnings and liquidity ratios. The Output consists of Fuzzy Values and defuzzified Values. The inference process has been implemented based on Fig.1. The rules of the inference process and certainty factors are represented in Tables 1, 2, 3, 4.

Fuzzy inference engine matches the financial fuzzy rules in the rules base with the entered values for the indicators data which is stored in the database to select which rules should be applied and manages the reasoning process.

\subsection{Working Memory}

Working memory is a repository for temporarily storing and managing the information required to carry out complex cognitive tasks such as learning, reasoning, and comprehension. Working memory in this proposed model is used to store everything that is related to the selection, initiation (rules firing), and termination of the inference operations and the information-processing functions such as encoding, storing, and retrieving data.

\subsection{DBMS}

A database management system (DBMS) allows bankers to perform model's database managing operations i.e. storing, retrieving, adding, deleting and modifying. 


\subsection{User Interface}

It allows communication between the system users (managers, bankers) and the implemented system of the model, and it is used to input the bank's data and receive the output.

\subsection{Database (DB)}

Database saves all the entered data about banks including the financial indicators data and it is managed by the DBMS which enables the users (managers and bankers) to add, update and delete any previously saved bank's data.

\subsection{Explanation Module}

The explanation module is responsible for explaining the results of the inference operation and to select the most appropriate advice for how the bank will remedy its financial problems that are related to the financial ratios used in bank failure prediction.

\subsection{Defuzzification Module}

A task of defuzzification is to map a fuzzy output to crisp output of the system. A number of defuzzification strategies exist. Each provides a means to choose a single output (which denoted as zj) based on the implied fuzzy sets. The input for defuzzification is the fuzzy set (the aggregate output fuzzy set) and the output is a crisp number. Defuzzification can be obtained by using three known ways:
a- The mean of maximum method
b- The maximizing decision.
c- The center of gravity method. [12]

In this paper, the center of gravity method is used as a defuzzification strategy.

\section{ALGORITHM OF THE PROPOSED MODEL}

This section presents an algorithm of the proposed model for predicting the financial failure of commercial banks.

1. Log in as a system admin.

2. Enter CBE indicators values for each ratio.

3. Calculate the membership function numerical range for each indicator linguistic value.

4. Log in as a bank manager.

5. If new bank

Enter financial indicators values and data of the bank.

Save financial indicators values and data of the bank.

Else

\{

Retrieve financial indicators values and data of the bank. \}

6. Decide the bank indicator membership value with the minimum matched calculated numeric range.

7. Calculate for each financial ratio, its final value by applying the appropriate fuzzy rules using the fuzzy inference engine.

8. Defuzzify the resulted calculated financial ratios values by applying the centroid equation.

9. Compare the defuzzification equation result with $\% 50$ (CBE's declared total bank performance evaluation threshold).

10. If financial failure
- $\quad$ Start to find the solutions and alternatives.

- $\quad$ Report the recommended solutions. \} Else

\{

- Generate bank performance report.

- Report the recommended solutions for indicators of the weakness.

\}

11. End.

Figure 2 shows the Activity Diagram of the Proposed Model.

\section{MODEL IMPLEMENTATION RESULTS DISCUSSION}

The proposed model uses fuzzy logic in MATLAB to calculate financial ratios membership functions such as capital adequacy member ship, asset quality member ship, earnings member ship and liquidity member ship. Fuzzy inference engine is based on four steps: fuzzification of the input variables, rule evaluation, aggregation of the rule outputs and defuzzification. Fuzzy logic output in MATLAB consists of two results: the first result is rules viewer and second result is surface viewer. The proposed model uses visual studio 2010 to make a graphical user interface (GUI) which is a type of user interface that allows bankers to interact with electronic devices with images rather than text commands. GUIs can be used in computers and hand-held devices. A GUI represents the information and actions available to a banker through graphical icons and visual indicators. The proposed model was implemented and applied on two Egyptian commercial banks (CIB, CFEB). This section presents and analyses the results of the implemented proposed model.

The calculated ratios by the model for the CIB bank are as follows:

1. Capital adequacy risk is low (8.75)

2. Asset Quality risk is medium (1.36)

3. Earnings risk is low (0.94)

4. Liquidity risk is low (13.9)

Calculation of CIB Bank failure percentage:

$\mathrm{Z}=$

$\frac{(8.75 * 25)+(1.36 * 50)+(0.94 * 25)+(13.9 * 25)}{8.75+1.36+0.94+13.9}$

$8.75+1.36+0.94+13.9$

$\mathrm{Z}=\frac{218.75+68+23.5+347.5}{24.95}$

$\mathrm{Z}=\frac{657.75}{24.95}=26.4$

Percentage of CIB bank failure by defuzzification is (26.4).

As a result, the proposed model predicted the percentage of financial failure of the CIB bank is 26.4 depending on capital adequacy risk is low (8.75), asset quality risk is medium (1.36), earnings risk is low (0.94) and liquidity risk is low (13.9). The implemented system advised as a remedy strategy for the CIB bank: (a) For the Asset Quality ratios: increase LPL indicator, and increase PA indicator. (b) For the Earnings ratios: increase NOIIA Indicator. (c) For the Liquidity ratios: increase SA Indicator. 


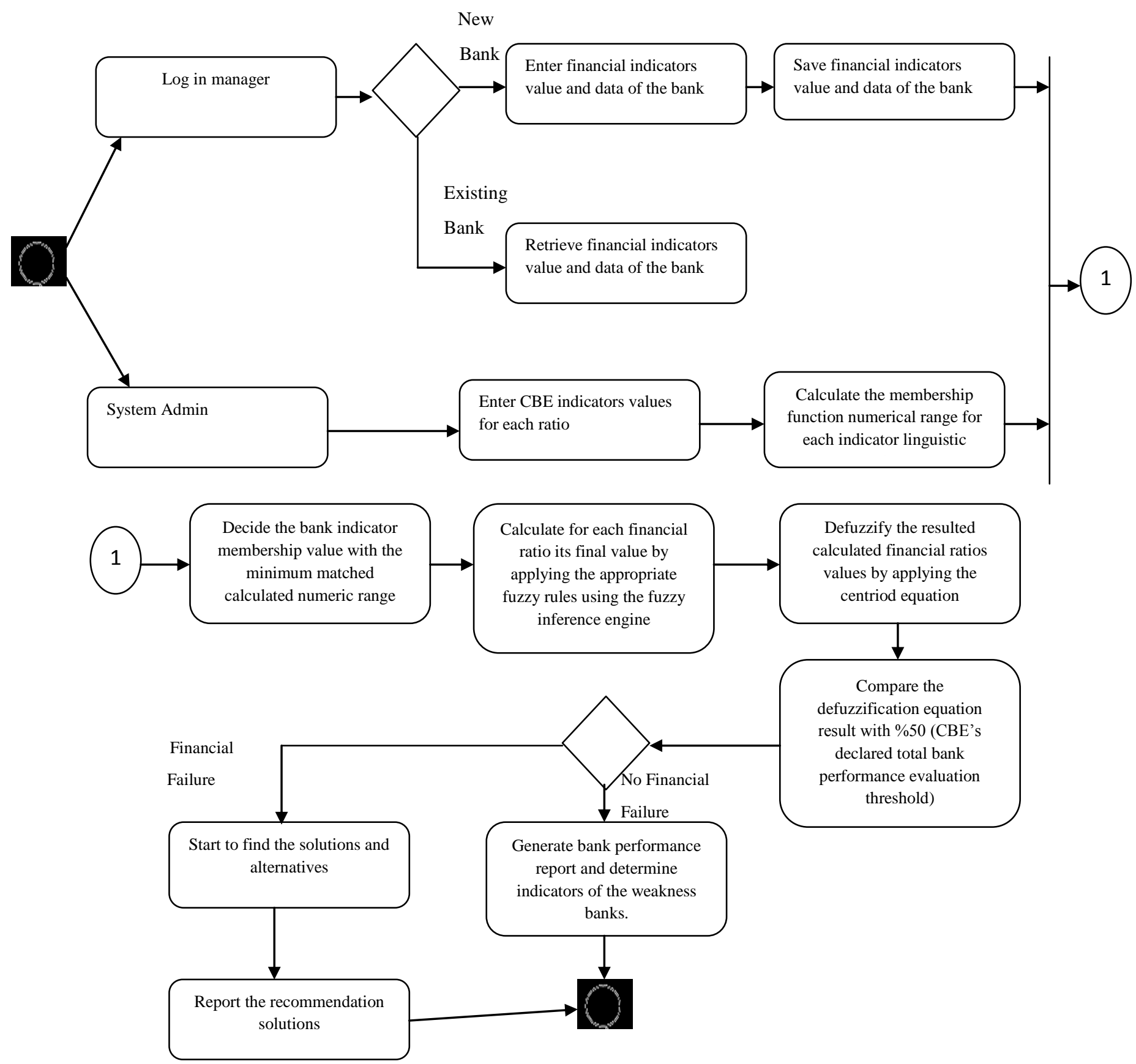

Fig 2: The Activity Diagram of the Proposed Model

The calculated ratios by the model for the CFEB bank are as follows:

1. Capital adequacy risk is low (3.9)

2. Asset Quality risk is medium (0.21)

3. Earnings risk is low (1.4)

4. Liquidity risk is low (7.5)

Calculation of CFEB Bank failure percentage:

$\mathrm{Z}=$

$$
\frac{(3.9 * 75)+(0.21 * 75)+(1.4 * 25)+(7.5 * 50)}{3.9+0.21+1.4+7.5}
$$

$\mathrm{Z}=\frac{292.5+15.75+35+375}{13.01}$

$$
13.01
$$

$\mathrm{Z}=\frac{718.25}{13.01}=55.2$

Percentage of CFEB bank failure by defuzzification is (55.2). As a result, the proposed model predicted the percentage of financial failure of the CFEB bank is 55.2 depending on that capital adequacy risk is low (3.9), asset quality risk is medium (0.21), earnings risk is low (1.4) and liquidity risk is low (7.5). The implemented system advised as a remedy strategy for the CFEB bank: (a) For the Capital Adequacy ratio: increase EA Indicator. (b) For the Asset Quality ratios: increase LPL Indicator, increase PA Indicator, and increase LPSTL Indicator. (c) For the Earnings ratios: increase NPE Indicator. 
(d) For the Liquidity ratios: increase SA Indicator, and increase DA Indicator.

\section{CONCLUSIONS}

The proposed model in this paper is successful in predicting the failure of the commercial banks in advance with a reasonable accuracy. After using the proposed model, bank managers will be able to determine the level of financial failure in commercial banks, and receive remedy advices for solving the bank financial problems and enhancing the financial indicators values. The technique of fuzzy logic is one of the most important machine learning techniques used to detect financial failure in commercial banks. After using the proposed model, fuzzy logic proved that it is scalable, reliable, stable, and different from Probability methods and based on natural language. For future work, it is advisable to integrate other machine learning techniques like artificial neural networks technique with the proposed fuzzy logic based model to enhance the results accuracy of the model.

\section{REFERENCES}

[1] Edward I. 1997 Financial Ratios, Discriminant Analysis and the Prediction of Corporate Bankruptcy, the Journal of Finance, Vol. 23, No. 4.pp. 589-609.

[2] Kolari, J., Glennon, D., Shin, H. and Caputo, M. 2000 Predicting Large U.S. Commercial Bank Failures, Economic and Policy Analysis Working Paper 2000-1, January.

[3] Laitinen, E.K. and Laitinen, T. 2000 "Bankruptcy prediction. Application of the Taylor's expansion in logistic regression", International Review of Financial Analysis. Vol.9, p372-349.
[4] Molina, C. A. 2002 Predicting bank failures using a hazard model: the Venezuelan banking crisis. Emerging Market Review 3, 31-50.

[5] Mete M., Başak, N., and Ramazan, C.2005 Predicting Financial Failure of the Turkish Banks, University, Ankara, Turkey.

[6] Lanine, G, and Vander R.2007 Failure prediction in the Russian bank sector, with logit and trait recognition models, Ghent University, Belgium, Ghent University, Belgium.

[7] Eygi B. 2008 Applied Mathematical Sciences, Vol. 2, no. 60, Marmara University, Art and Science Faculty, Department of Mathematics, Goztepe, Istanbul, Turkey, $2973-2982$.

[8] Maisarh, F., Yaseen, M., and Omar, N. 2008 Predicting Financial Failure of Yemeni Banks, College of Business, Division of Finance and Banking, University Utara Malaysia.

[9] Mohammed, N. 2010 "Exploiting Machine Learning in Generating Future scenarios for the Tourism Industry in Egypt", Cairo University, Faculty of Computers and Information.

[10] Dostál, P. 2013 Forecasting of Time Series with Fuzzy Logic. Advances in Intelligent Systems and Computing, Volume 210, pp 155-161.

[11] Andrew G., Belik, I., and Rahimi1, S. 2010 "A Hybrid Expert System for IT Security Risk Assessment", Southern Illinois University, Carbondale, IL, USA. 